\title{
A non-hydrostatic and compressible 2-D model simulation of Internal Gravity Waves generated by convection
}

\author{
Kenshi Goya and Saburo Miyahara \\ Department of Earth and Planetary Sciences, Faculty of Science, Kyushu University, Hakozaki 6-10-1, Fukuoka 812-8581, Japan
}

(Received August 4, 1998; Revised January 28, 1999; Accepted April 9, 1999)

\begin{abstract}
Generation of internal gravity waves (IGWs) by tropospheric convections and vertical propagation of the generated IGWs throughout the middle atmosphere are simulated by a non-hydrostatic compressible nonlinear two-dimensional numerical model.

The present simulation demonstrates that (i) IGWs are generated by the tropospheric dry convections, (ii) zonal mean wind is decelerated by critical layer absorption of the generated IGWs in the upper tropospheric shear zone, (iii) secondary IGWs are radiated by the critical layer instability, and (iv) the secondary IGWs break down and accelerate zonal mean winds in the upper middle atmosphere.

The detailed analyses show that (1) eastward propagating IGWs generated by the convection in tropospheric westerly with small wavelength of the order of $10 \mathrm{~km}$ and short period of the order of 10 min are dominant. It is found that the dominant waves are selected by a filtering effects of the prescribed westerly. (2) Several secondary IGWs with smaller horizontal wavelength than the primary IGW are radiated. The secondary IGWs propagate vertically in the form of wavepackets and break in the upper middle atmosphere due to local convective instability because of the exponential growth of the wave amplitudes with height. In the breaking region, the observed and theoretically predicted universal power law of the wind fluctuation, which states the $m^{-3}$ dependence for the power spectra versus the vertical wavenumber $m$ due to the wave saturation and breakdown, is also realized in the present model simulation.
\end{abstract}

\section{Introduction}

It has been widely recognized that Internal Gravity Waves (IGWs) play an important role in the middle atmosphere dynamics, since the studies of Lindzen (1981) and Matsuno (1982) appeared. Behaviors of IGWs propagation in the atmosphere, wave/mean flow and critical-level interaction with IGWs, breakdown of IGWs, and radiation of secondary IGWs from the breakdown region are interesting issues in the atmospheric dynamics related to the IGWs.

The first detailed analysis of the critical-level interaction of an IGW with mean flow was conducted by Bretherton (1966) and Booker and Bretherton (1967). These authors used a linear inviscid Boussinesq approximation to describe both the steady state and the transient behavior of an IGW near the critical level, and proved that the interaction at the critical level results in a gravity wave attenuation. It was demonstrated that much of the energy and momentum of the wave are transferred to the mean flow near the critical level. However, such steady solutions are never realized in the atmosphere, so that studies with time dependent and nonlinear models were required to investigate realistic behaviors of IGWs in the atmosphere.

Using a nonlinear incompressible two-dimensional model, Geller et al. (1975) showed that IGWs become unstable near the critical level and produce turbulence in the unstable re-

Copy right $(\mathrm{C}$ The Society of Geomagnetism and Earth, Planetary and Space Sciences (SGEPSS); The Seismological Society of Japan; The Volcanological Society of Japan; The Geodetic Society of Japan; The Japanese Society for Planetary Sciences. gion. However they did not show the subsequent behavior of IGWs after the instability. It was found by Fritts (1979) using a time-dependent two-dimensional Boussinesq model that the gravity wave-critical level interaction can induce both the Kelvin-Helmholtz instabilities and the radiation of secondary waves through nonlinear interactions near the critical level.

It was suggested by Hodges (1967) that sporadic breakdown of IGWs is an important source of turbulence which can produce sufficient eddy diffusion to explain the thermal structure of the mesopause region. Fritts (1982) conducted a time dependent nonlinear numerical simulation of breakdown of IGWs and showed that IGWs break down due to the convective instability associated with the large amplitude resulting in the turbulent dissipation of the incident IGWs. Fritts et al. (1994) recently reported the detailed evolution of the breakdown of IGWs and the generation of mean flow using a time dependent nonlinear three-dimensional model, in which they focused their attention to the IGW breaking phenomena.

IGWs are thought to be generated by the topography, thermal convections, fronts, shear instability etc. In particular IGWs generated by the topography are believed to deposit most momentum and to produce weak zonal wind structure in the tropopause region (e.g., Palmer et al., 1986; McFarlane, 1987). Topographic sources are thought to be localized, while convections in the troposphere are ubiquitous. According to the observational report by aircrafts and ST radar sampling of wind and temperature fluctuation (Fritts and 
Nastrom, 1992), it is deduced that the convective sources may be important for the tropics and southern hemisphere where there are few topographic sources. Sato (1997) suggested that convections have the ability to generate short period $(<1$ day) gravity waves with small horizontal scales of the order of from 10 to $100 \mathrm{~km}$. Thus the propagation of IGWs generated by convections in the troposphere is of interest to investigate. Forvell et al. (1992) simulated vertical propagation of IGWs excited by a squall line below the lower stratosphere in a nonhydrostatic compressible two-dimensional numerical model. Using the similar model, Alexander et al. (1995) investigated forcing mechanism of IGWs due to a simulated squall line. However, upward propagation of IGWs into the upper middle atmosphere is not simulated in their model. Prusa et al. (1996) simulated propagation and breaking of IGWs in the upper middle atmosphere, which were excited by a lower boundary forcing at the troposphere, using a non-hydrostatic compressible two-dimensional numerical model.

In order to elucidate dynamical behaviors and evolution of high frequency and small scale IGWs throughout the middle atmosphere generated by tropospheric convections, a nonhydrostatic and compressible numerical model is required. In this study, we develop a non-hydrostatic compressible nonlinear two-dimensional model and simulate the generation of IGWs by convections in the troposphere, and simulate the propagation and the breakdown of the IGWs in the middle atmosphere. The present model also simulates the radiation and propagation of secondary waves due to the breakdown of the incident IGWs.

This paper is organized in the following manner. The model description is done in Section 2. The results and discussions are described in Section 3. The conclusions and remarks are presented in Section 4.

\section{Description of the Model}

\subsection{Basic equation system}

A non-hydrostatic compressible two-dimensional nonlinear numerical model is developed to simulate convective instability in the troposphere, generation of IGWs in the convective region, upward propagation of the generated IGWs, breakdown of the propagating IGWs, and radiation of secondary IGWs from the breaking region. The equation system is expressed as follows (e.g., Forvell et al., 1992):

$$
\begin{gathered}
\frac{\partial \tilde{\delta u}}{\partial t}=-\frac{\partial u \tilde{u}}{\partial x}-\frac{\partial w \tilde{u}}{\partial z}-\frac{\partial p}{\partial x}-K_{\mathrm{H}} \frac{\partial^{4} \tilde{\delta u}}{\partial x^{4}}+K_{\mathrm{V}} \frac{\partial^{2} \tilde{\delta u}}{\partial z^{2}}-R_{\mathrm{f}} \tilde{\delta u}, \\
\frac{\partial \tilde{\delta w}}{\partial t}=-\frac{\partial u \tilde{w}}{\partial x}-\frac{\partial w \tilde{w}}{\partial z}-\frac{\partial p}{\partial z}-\rho g \\
-K_{\mathrm{H}} \frac{\partial^{4} \tilde{\delta w}}{\partial x^{4}}+K_{\mathrm{V}} \frac{\partial^{2} \widetilde{\delta w}}{\partial z^{2}}-R_{\mathrm{f}} \widetilde{\delta w} \\
\frac{\partial \widetilde{\delta T}}{\partial t}=-\frac{\partial u \widetilde{T}}{\partial x}-\frac{\partial w \widetilde{T}}{\partial z}-\frac{R \widetilde{T}}{c_{\mathrm{V}}}\left(\frac{\partial u}{\partial x}+\frac{\partial w}{\partial z}\right) \\
-K_{\mathrm{H}} \frac{\partial^{4} \widetilde{\delta T}}{\partial x^{4}}+K_{\mathrm{V}} \frac{\partial^{2} \tilde{\delta T}}{\partial z^{2}}-\alpha \widetilde{\delta T}, \\
\frac{\partial \rho}{\partial t}+\frac{\partial \tilde{u}}{\partial x}+\frac{\partial \tilde{w}}{\partial z}=0,
\end{gathered}
$$

$$
p=R \widetilde{T},
$$

where $u$ denotes horizontal wind, $w$ vertical wind, $T$ temperature, $\rho$ density, $p$ pressure, $g$ gravitational acceleration, $R$ gas constant for dry air, $c_{\mathrm{V}}$ specific heat of dry air at constant volume, $K_{\mathrm{H}}$ horizontal eddy diffusion coefficient, $K_{\mathrm{V}}$ vertical eddy diffusion coefficient, $R_{\mathrm{f}}$ Rayleigh friction coefficient, and $\alpha$ Newtonian cooling coefficient.

The product of the dynamical variables $[u, w, T]$ and $\rho$ denoted by the tilde, for example,

$$
\tilde{u}(x, z, t) \equiv u(x, z, t) \cdot \rho(x, z, t) .
$$

Furthermore the deviation of the each variable from an initial basic state, which is independent of time, is denoted by $\delta$ as follows:

$$
\begin{array}{r}
\tilde{\delta u}(x, z, t)=\tilde{u}(x, z, t)-\tilde{u}_{\mathrm{B}}(z), \quad \tilde{u}_{\mathrm{B}}(z) \equiv \rho_{\mathrm{B}}(z) u_{\mathrm{B}}(z), \\
\tilde{\delta w}(x, z, t)=\tilde{w}(x, z, t), \\
\widetilde{\delta T}(x, z, t)=\widetilde{T}(x, z, t)-\widetilde{T}_{\mathrm{B}}(z), \quad \widetilde{T}_{\mathrm{B}}(z) \equiv \rho_{\mathrm{B}}(z) T_{\mathrm{B}}(z),
\end{array}
$$

where the subscript B denotes the initial basic state.

\subsection{Spectral and finite difference presentation of vari- able}

The physical variables are expanded in the horizontal direction by the complex Fourier series as follows,

$$
\tilde{\delta a}(x, z, t)=\sum_{k=-K M}^{K M} \tilde{\delta a}_{k}(z, t) \exp (i k x), \quad \tilde{\delta a}_{k}=\left(\tilde{\delta a}_{-k}\right)^{*}
$$

where $k$ denotes horizontal wavenumber, $K M$ the maximum horizontal wavenumber, and the superscript $*$ denotes the complex conjugate. In the vertical direction, a finite difference method is used, and the variables are located on the

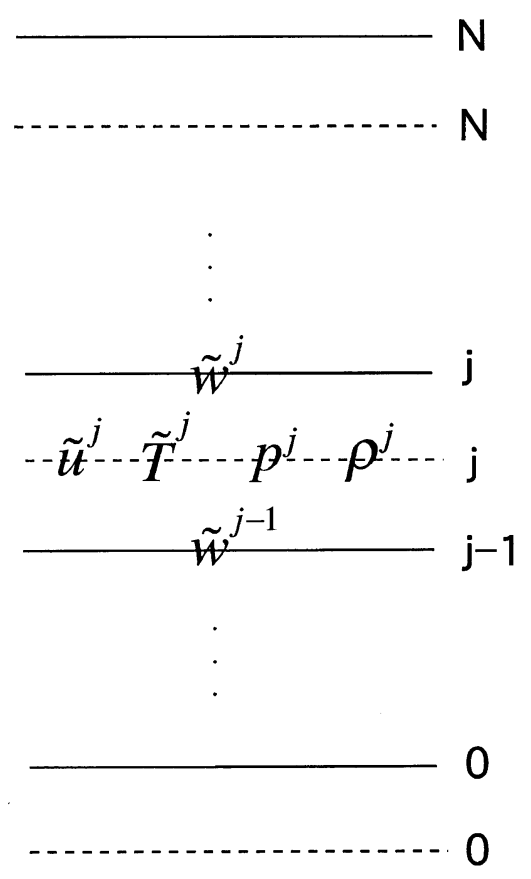

Fig. 1. Variables collocations. Superscript j denotes vertical level number. 

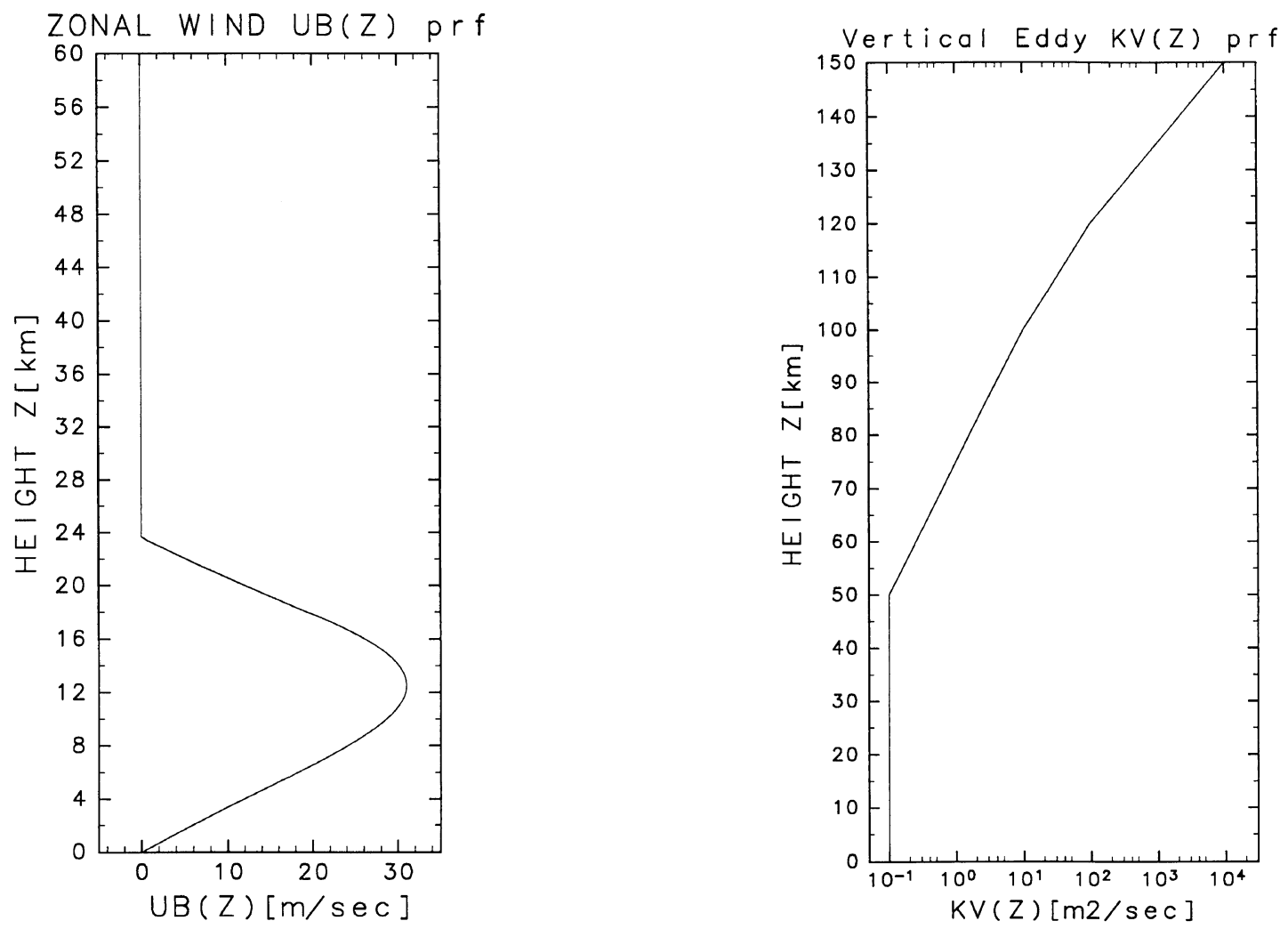

Fig. 2. Vertical profile of initial background horizontal wind $U_{\mathrm{B}}(z)\left(\mathrm{m} \mathrm{s}^{-1}\right)$.

Fig. 4. Vertical profile of vertical eddy diffusion coefficient $K_{\mathrm{V}}(z)\left(\mathrm{m}^{2} \mathrm{~s}^{-1}\right)$.

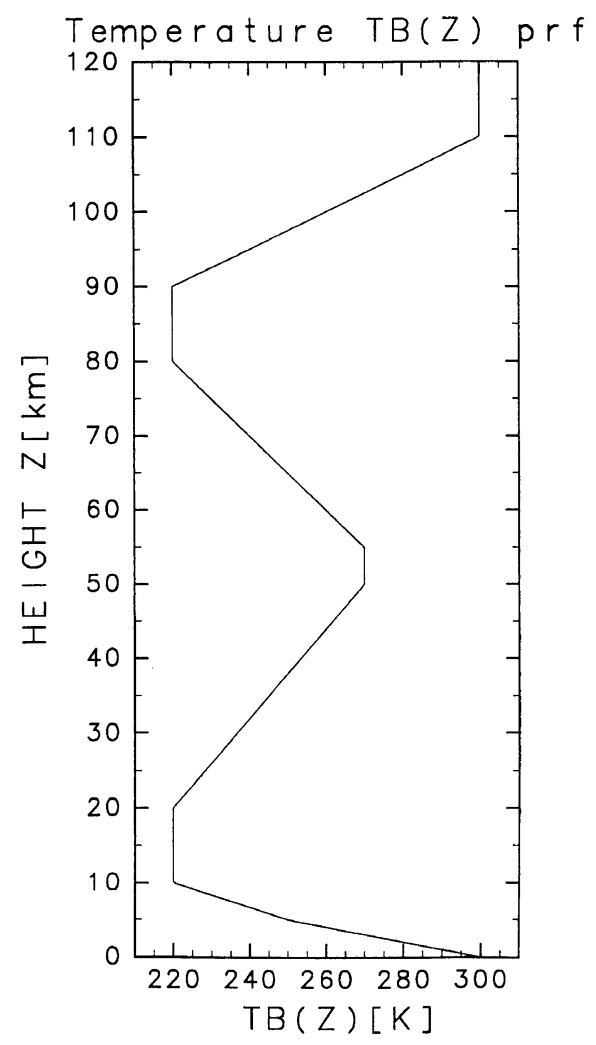

Fig. 3. Vertical profile of initial background temperature $T_{\mathrm{B}}(z)(\mathrm{K})$.

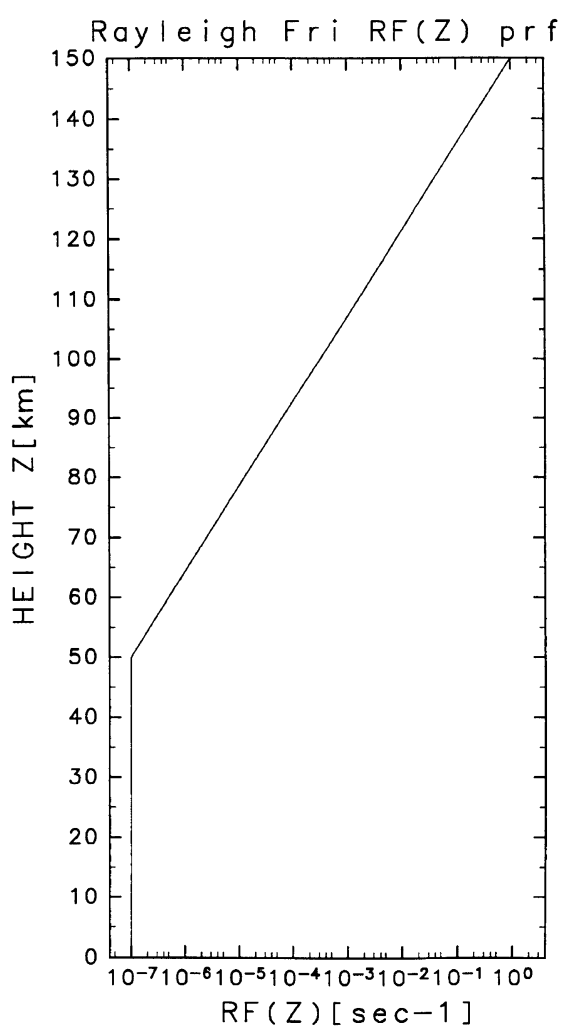

Fig. 5. Vertical profile of Rayleigh friction coefficient $R_{\mathrm{f}}(z)\left(\mathrm{s}^{-1}\right)$. 


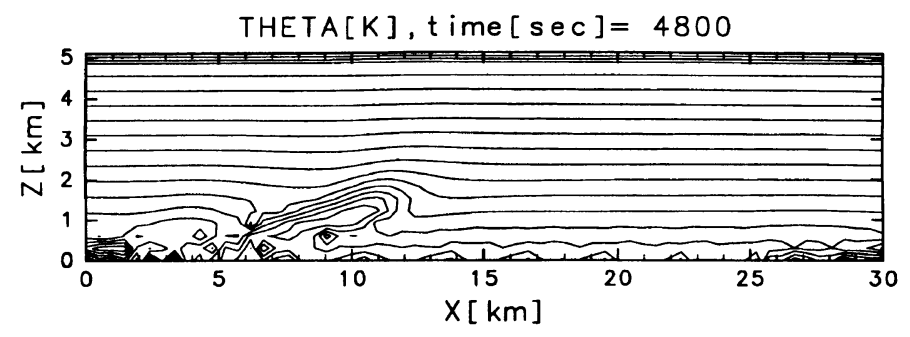

CONTOUR INTERVAL $=1.000 E-01$
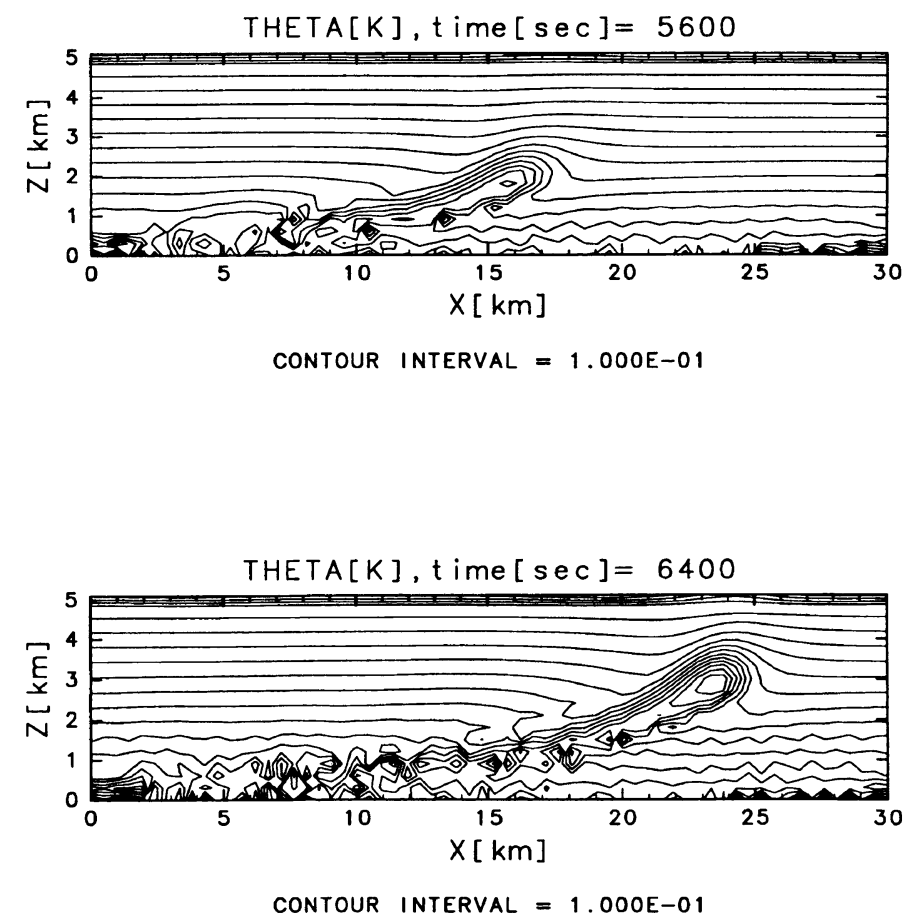

(a)

Fig. 6. Contour plots of (a) potential temperature (K) (below $5 \mathrm{~km}$ height) (b) $u^{\prime}\left(\mathrm{m} \mathrm{s}^{-1}\right)$ (deviation from zonal mean wind ) (c) $w$ (m s$\left.{ }^{-1}\right)$ (vertical wind), at the time steps of $4,800 \mathrm{~s}, 5,600 \mathrm{~s}$, and $6,400 \mathrm{~s}$. Negative values are shaded.

staggered grid, as shown in Fig. 1. Time integrations are performed in the wavenumber space. However, the nonlinear terms, the vertical diffusion terms are evaluated at every grid point using the grid point values of the physical variables, and they are transformed into the wave space. The transformations from the wavenumber space to the real space and vice versa are accomplished by the fast Fourier transform (FFT) with 128 grid points.

\subsection{Time integration}

The leap-frog scheme is used for the time-integration in the present model. Reinitialization using the simple forward scheme is applied every five time steps. For the dissipation terms, the simple forward scheme is used to keep the time integration stable. The time step is chosen to be $0.1 \mathrm{sec}$. To save the CPU time, nonlinear flux divergence terms in (1a), (1b) are evaluated every twenty time steps using the method mentioned above.

\subsection{Resolution}

The full model domain is chosen to be $30 \mathrm{~km}$ in the horizontal direction and in the vertical direction $150 \mathrm{~km}$ from the ground to the top of the model. The maximum horizontal wavenumber $K M$ is chosen to as 42 , so that the cutoff horizontal wavelength is $0.71 \mathrm{~km}$. $\Delta z$ is chosen to be $0.3 \mathrm{~km}$. It is confirmed that similar results to the present simulation are obtained when $\Delta z$ is chosen to be smaller value $0.15 \mathrm{~km}$.

\subsection{Basic state}

Figures 2 and 3 show the assumed initial basic states $U_{\mathrm{B}}(z)$ and $T_{\mathrm{B}}(z)$, respectively. $U_{\mathrm{B}}(z)$ is chosen to represent the vertical profile of zonal mean wind in the troposphere and lower stratosphere at middle latitude, but the values higher than 24 $\mathrm{km}$ height are assumed to be zero in order to see the wave propagation clearly. $T_{\mathrm{B}}(z)$ is chosen to represent the realistic temperature profile of the atmosphere, but the values lower than $5 \mathrm{~km}$ height are tuned to generate convective instability 


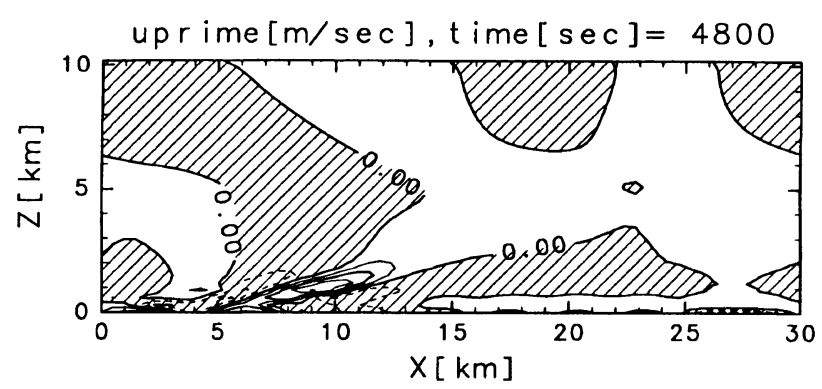

CONTOUR INTERVAL $=5.000 E-01$

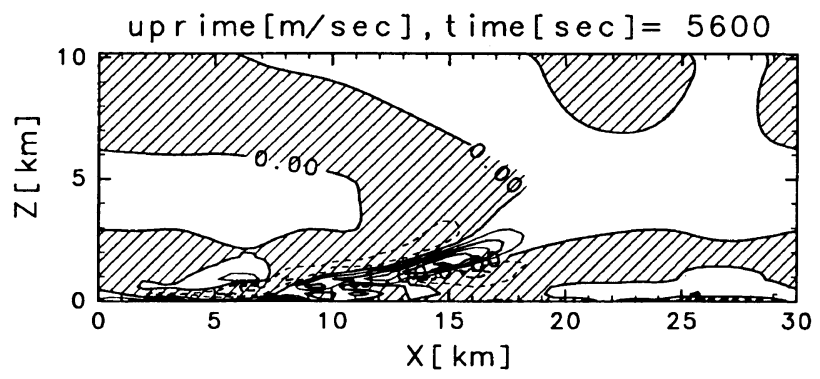

CONTOUR INTERVAL $=5.000 E-01$

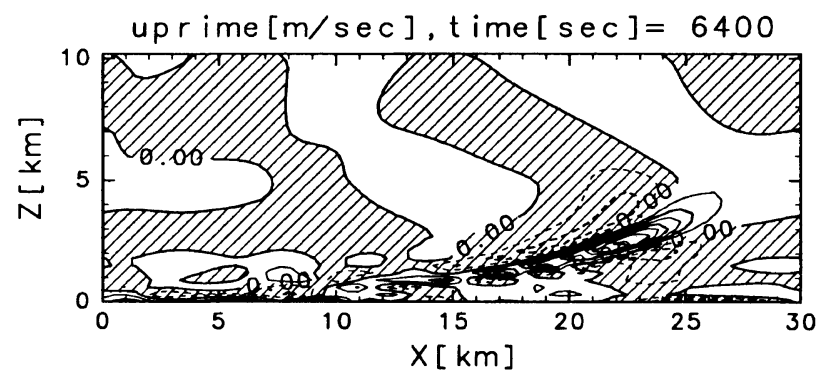

CONTOUR INTERVAL $=5.000 E-01$

(b)
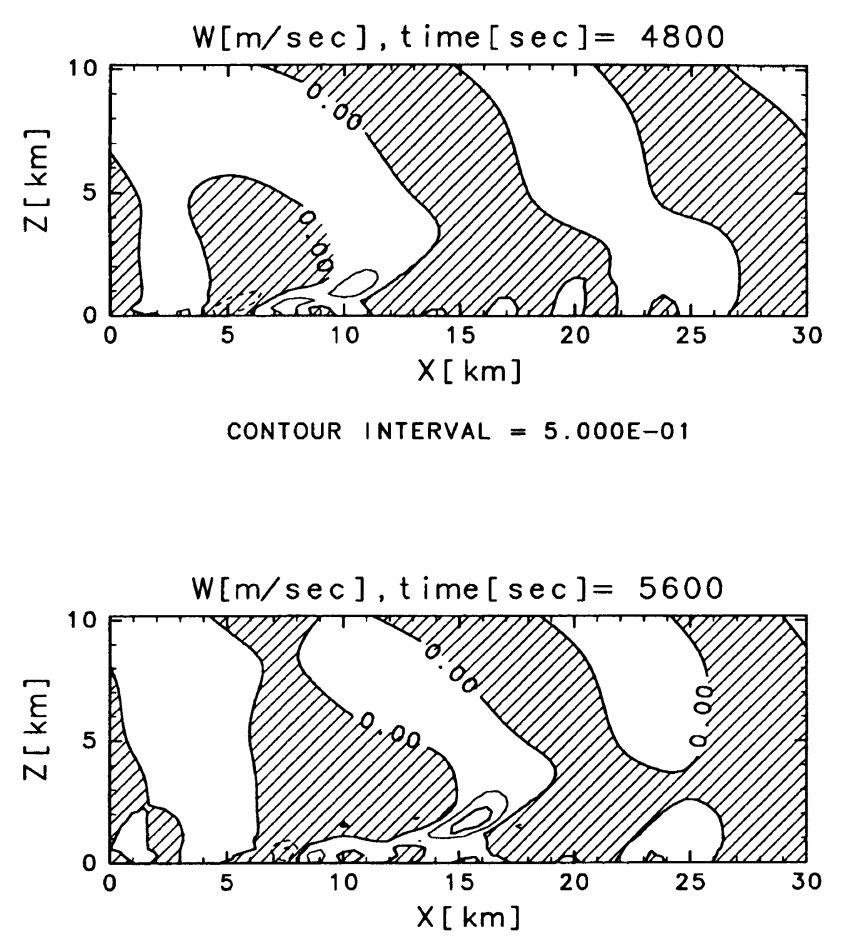

CONTOUR INTERVAL $=5.000 E-01$

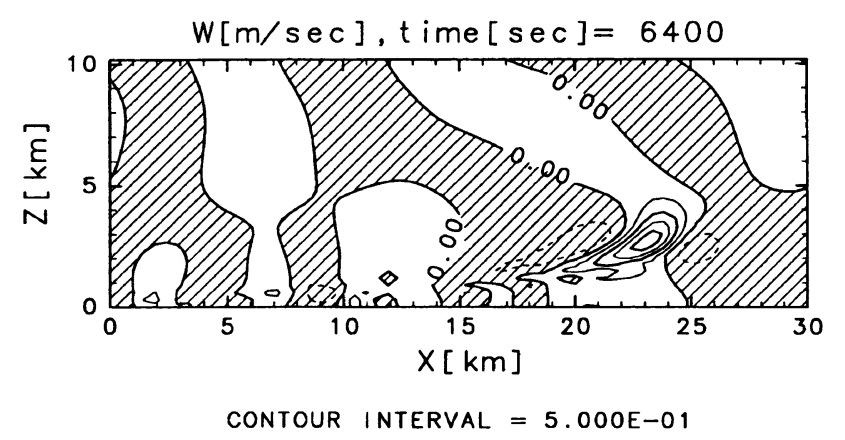

(c)

Fig. 6. (continued).

choosing the lapse rate to be $d T_{\mathrm{B}}(z) / d z=-10 \mathrm{~K} \mathrm{~km}^{-1}$. The values higher than $110 \mathrm{~km}$ are assumed to be constant $300 \mathrm{~K}$. This assumption is used to avoid extremely fast sound speed in the thermosphere which makes the numerical simulation difficult. The bottom temperature is fixed as $300 \mathrm{~K}$, thus the convectively unstable condition near the surface is always kept, and the convective motions are continuously generated in the course of the time integration.

\subsection{Initial and boundary conditions}

All variables to be time-integrated are initially set to be zero, except for $\widetilde{\delta w}$ at the bottom,

$$
\tilde{\delta u}=0, \quad \widetilde{\delta w}=0, \quad \widetilde{\delta T}=0, \quad \delta \rho=0, \quad \delta p=0 .
$$

$\widetilde{\delta w}$ at the bottom is given initially by white noise of the amplitude of $10^{-5} \mathrm{~m}^{-2} \mathrm{~kg} \mathrm{~s}^{-1}$ for all wavenumbers, which give the convection seeds. The boundary condition at the top is set to be zero,

$$
\tilde{\delta u}^{N}=0, \quad \widetilde{\delta w}^{N}=0, \quad \widetilde{\delta T}^{N}=0
$$

The bottom condition is given as follows. It is assumed that the vertical derivatives of $\widetilde{\delta u}$ and $\widetilde{\delta T}$ is equal to zero, and also assumed that the vertical velocity becomes zero, thus,

$$
\tilde{\delta u}^{0}=\tilde{\delta u}^{1}, \quad \widetilde{\delta w}^{0}=0, \quad \widetilde{\delta T}^{0}=\widetilde{\delta T}^{1}
$$

The condition at the lateral boundaries is assumed to be cyclic. This condition is realized by the Fourier expansion mentioned before.

\subsection{Dissipation}

Figures 4 and 5 show the vertical eddy diffusion coefficient $K_{\mathrm{V}}(z)$ and the Rayleigh friction coefficient $R_{\mathrm{f}}(z)$ used in the present simulation, respectively. The Newtonian cooling 


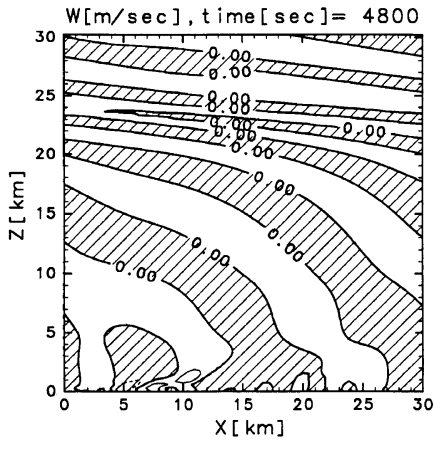

CONTOUR INTERVAL $=5.000 E-01$

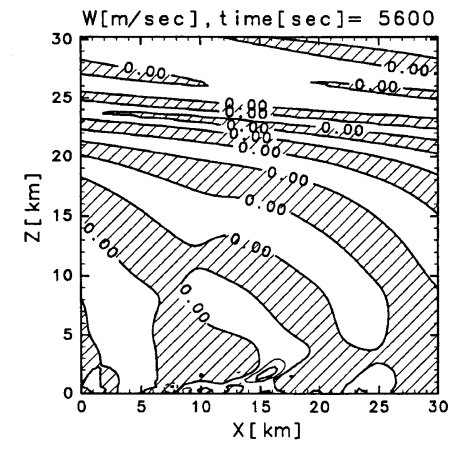

CONTOUR INTERVAL $=5.000 E-01$

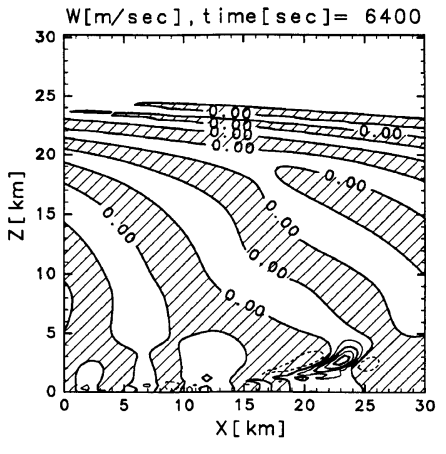

CONTOUR INTERVAL $=5.000 E-01$

Fig. 7. As in Fig. 6(c), except for the plots below $30 \mathrm{~km}$ height.
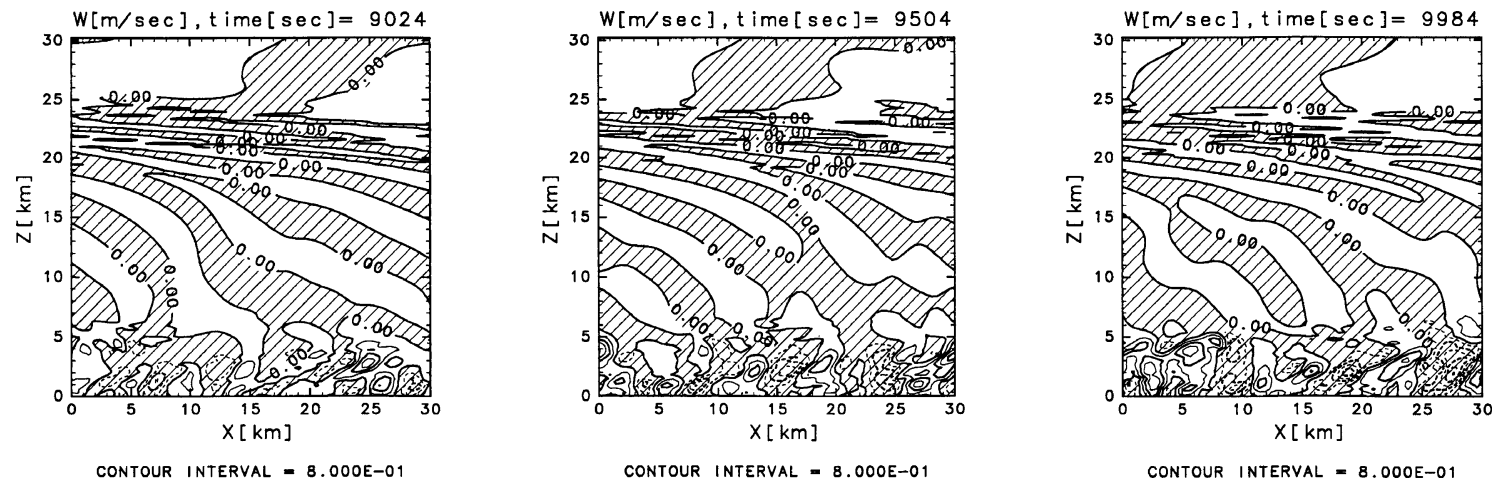

Fig. 8. As in Fig. 7, except for the time steps of 9,024 s, 9,504 s, and 9,984 s.

coefficient $\alpha(z)$ is assumed to be the same value with $R_{\mathrm{f}}(z)$. They are given to prevent the spurious reflection from the top boundary. An uniform horizontal eddy diffusion coefficient $K_{\mathrm{H}}$ of the value of $8.0 \times 10^{7} \mathrm{~m}^{4} \mathrm{sec}^{-1}$ is used to prevent a spurious energy accumulation at the truncated wavenumber. The correct vertical diffusion scheme, for instance in (1a), is $\frac{\partial}{\partial z}\left(\rho K_{\mathrm{V}} \frac{\partial u}{\partial z}\right)$. However, the simplified form shown in (1a) is used for simplicity in the present model. The difference of these two scheme is negligibly small for the parameter range in the present numerical simulation. Effects of eddy diffusion below $100 \mathrm{~km}$ in the numerical results are negligibly small.

\section{Results and Discussions}

\subsection{Convection and IGWs propagation}

Figures 6(a), (b) and (c) show the cross section of the total potential temperature, the horizontal wind fluctuation from the zonal mean, and the vertical wind in the troposphere, respectively, at the time steps of 4,800 s, 5,600 s, and 6,400 s. It is found that a disturbance having overturning potential temperature structure grows with time and moves eastward with the velocity which is almost equal to the background horizontal wind velocity at the height where the center of the disturbance is located. Compared with Figs. 6(b) and (c), it is found that the potential temperature disturbance corresponds to the strongest region of the horizontal and the vertical wind fluctuations. Other than the strongest disturbance, there are a few pairs of weaker convective cells. Figures 6(b) and (c) show that all these cells also move eastward almost with the same velocity of the background horizontal wind at the height where the centers of the cells are located. The movement of the convective cells is consistent with the linear analysis by Asai (1970) which showed that the unstable mode of the thermal convection travels at the velocity of the basic vertical shear flow averaged over the entire depth of the unstable layer. As shown in Figs. 6(b) and (c), the maximum magnitude of the horizontal wind fluctuation is about $3 \mathrm{~m} \mathrm{~s}^{-1}$ and the maximum vertical wind is about $2 \mathrm{~m} \mathrm{~s}^{-1}$ at these time steps.

Above the convectively unstable region, the westward tilted wave pattern with the horizontal wavelength of about $10 \mathrm{~km}$ is seen. In order to see the wave pattern in the above layer more clearly, the vertical wind distributions below 30 $\mathrm{km}$ at the same time steps of Fig. 6(c) are shown in Fig. 7. According to a space-time spectral analysis for the time span from $4,800 \mathrm{~s}$ to $6,848 \mathrm{~s}$ at $z=12 \mathrm{~km}$, it is found that the horizontal wavelength of a dominant wave is $10 \mathrm{~km}$ and the period is $35 \mathrm{~min}$ and the wave is moving eastward with the phase velocity of $4.8 \mathrm{~m} \mathrm{~s}^{-1}$. Taking into account the Doppler effect by the zonal mean wind of $30 \mathrm{~m} \mathrm{~s}^{-1}$ at $12 \mathrm{~km}$ height, it is found that the intrinsic period of the wave is $6.6 \mathrm{~min}$ and the wave is moving westward relative to the zonal mean wind. The vertical wavelength of the dominant wave is deduced to be about $8 \mathrm{~km}$ in the vertical range from $10 \mathrm{~km}$ to 


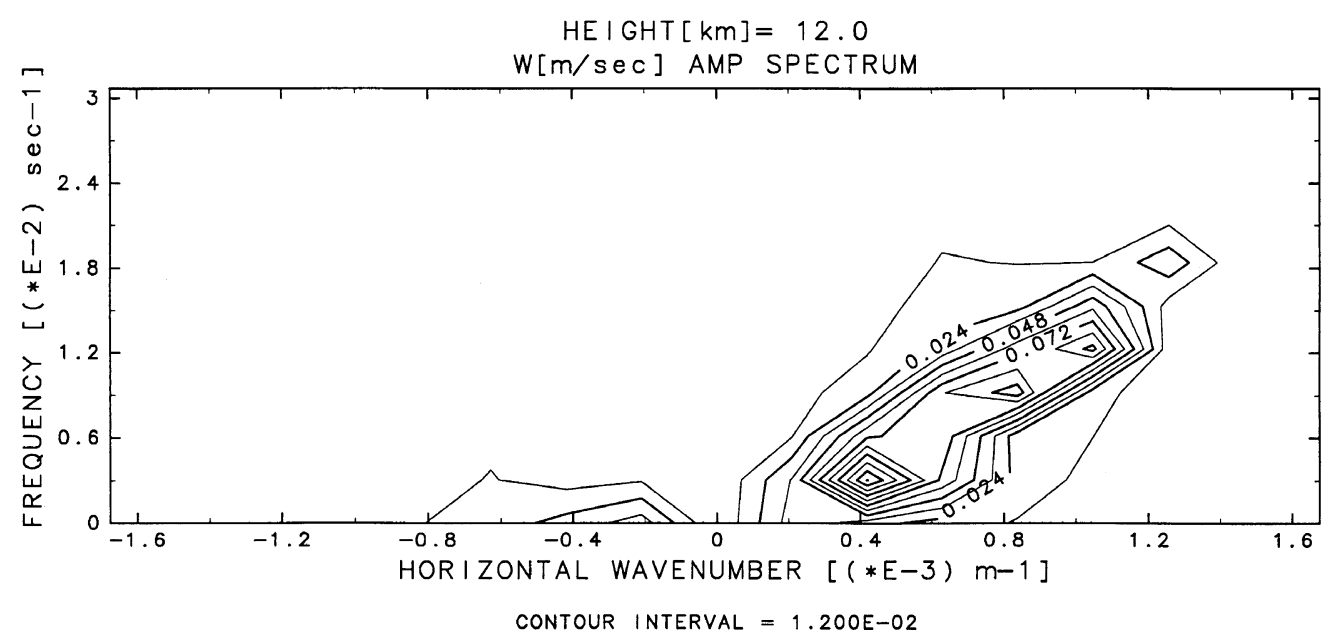

Fig. 9. Space-time spectrum of the vertical wind $w\left(\mathrm{~m} \mathrm{~s}^{-1}\right)$ at $12 \mathrm{~km}$ height for the time span from $8,000 \mathrm{~s}$ to $10,048 \mathrm{~s}$. Positive horizontal wavenumber denotes eastward moving and negative denotes westward moving components.

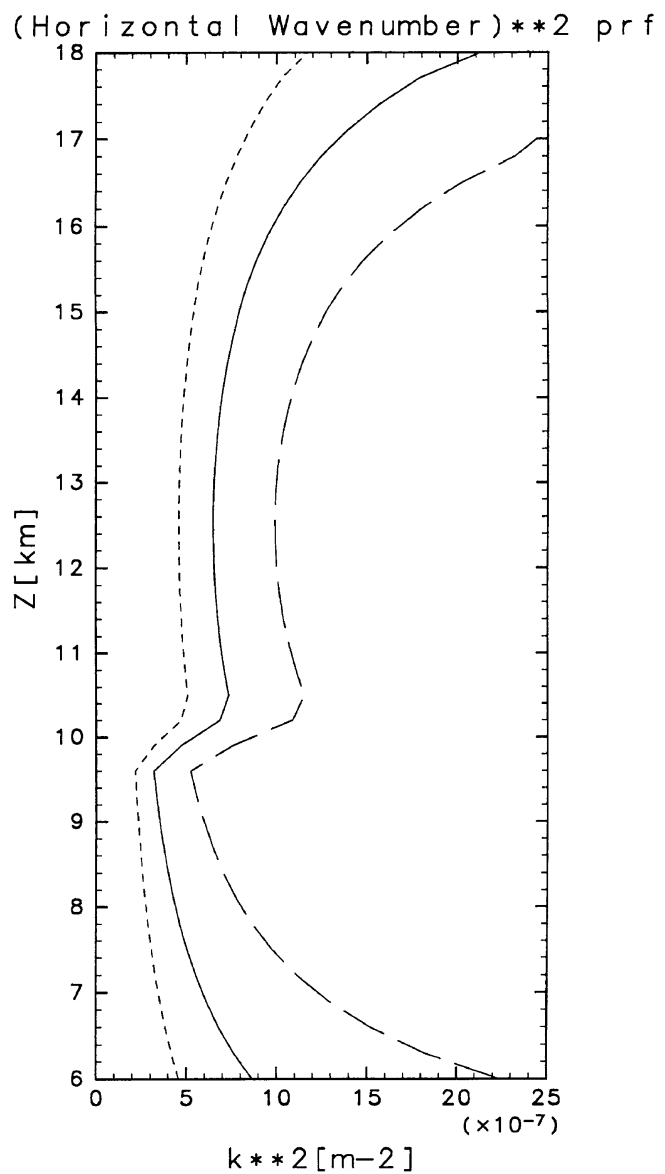

Fig. 10. Vertical profile of square of horizontal wavenumber $k^{2}$ associated with waves of $m^{2}=0$ for phase velocity $c=5 \mathrm{~m} \mathrm{~s}^{-1}$ (solid), $10 \mathrm{~m} \mathrm{~s}^{-1}$ (dashed), $2.5 \mathrm{~m} \mathrm{~s}^{-1}$ (dotted).

$20 \mathrm{~km}$ height. These parameters of the wave approximately satisfy the following dispersion relation of IGWs assuming the mean temperature $\bar{T}=250 \mathrm{~K}$,

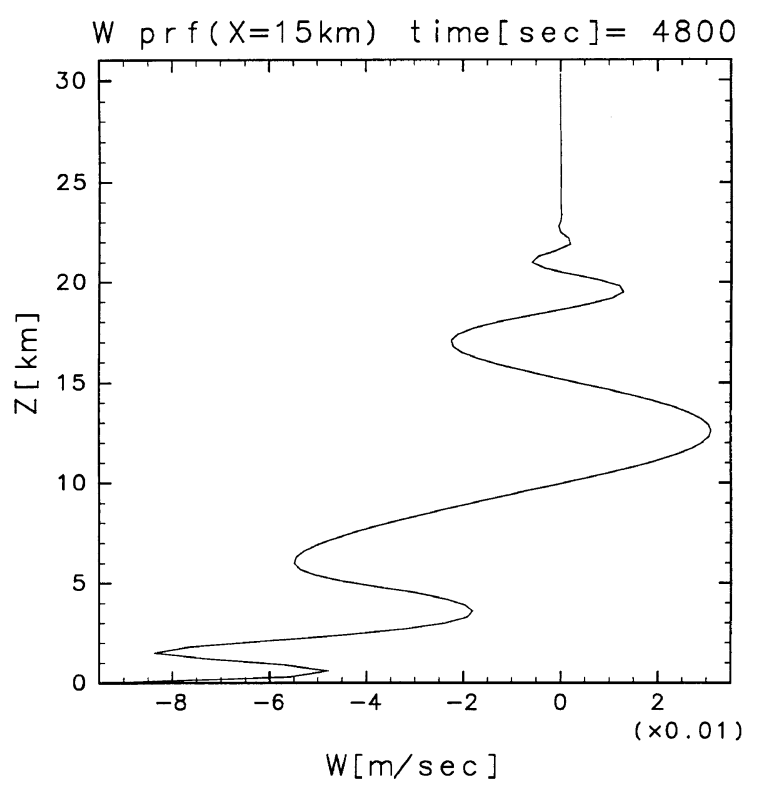

Fig. 11. Vertical profile of vertical wind $\left(\mathrm{m} \mathrm{s}^{-1}\right)$ at $4,800 \mathrm{~s}$.

$$
\hat{\omega}^{2}=\frac{N^{2} k^{2}}{k^{2}+m^{2}+\frac{1}{4 H^{2}}}, \quad \hat{\omega} \equiv \omega-\bar{U} k=(c-\bar{U}) k,
$$

where $\hat{\omega}$ denotes the intrinsic frequency, $\omega$ frequency, $k$ horizontal wave number, $m$ vertical wave number, Burant-Väisällä frequency, and the scale height is defined by $H \equiv R \bar{T} / \mathrm{g}$. Thus the wave generated by the convection is identified as an IGW.

Figure 8 shows the cross section of vertical wind at the time steps of 9,024 s, 9,504 s, and 9,984 s. The structure in the convective layer become more complicated as time goes on. The space-time spectral analysis for the time span from $8,000 \mathrm{~s}$ to $10,048 \mathrm{~s}$ at $12 \mathrm{~km}$ height indicates the existence of eastward moving waves with larger horizontal wavenumber 


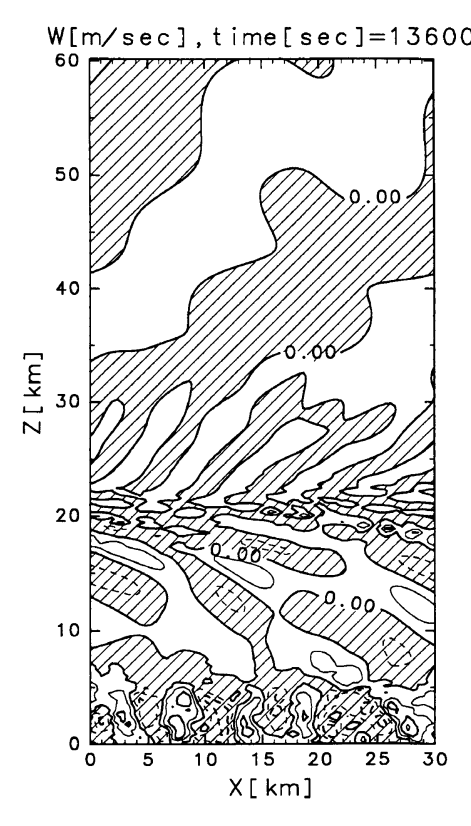

CONTOUR INTERVAL $=1.000 E+00$

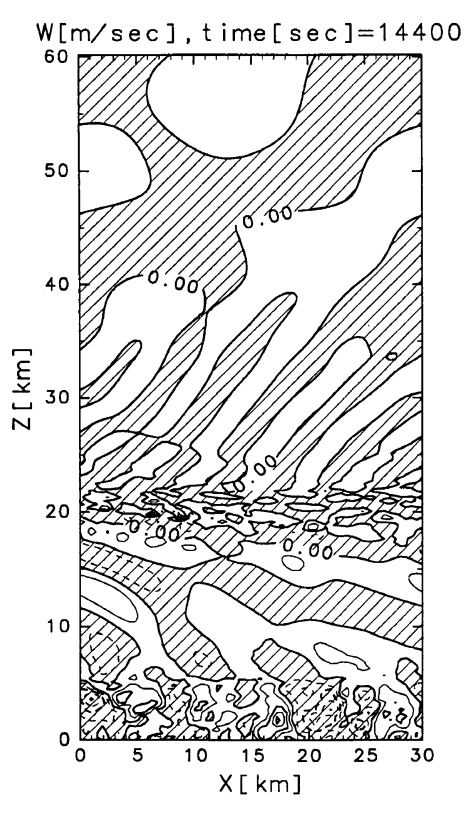

CONTOUR INTERVAL $=1.000 E+00$

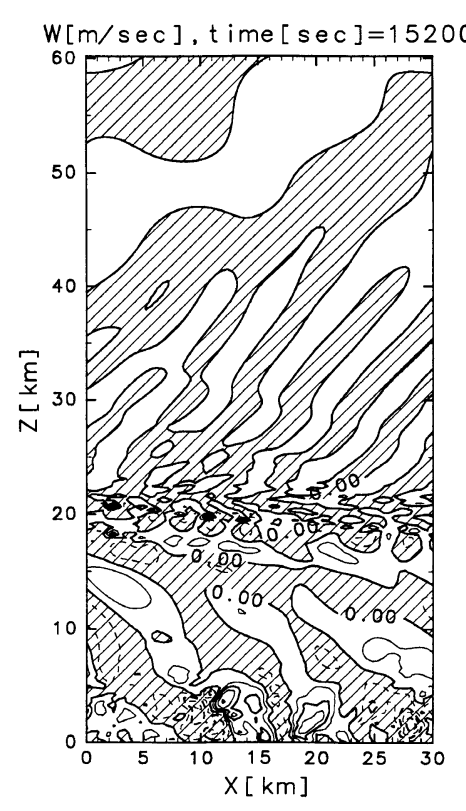

CONTOUR INTERVAL $=1.000 E+00$

Fig. 12. Contour plots of vertical wind $w\left(\mathrm{~m} \mathrm{~s}^{-1}\right)$ below $60 \mathrm{~km}$ height at the time steps of 13,600 s, 14,400 s, and 15,200 s. Negative values are shaded.

and higher frequency spectrum as shown in Fig. 9 than the IGW shown in Fig. 7. The phase velocities of the waves are about $10 \mathrm{~m} \mathrm{~s}^{-1}$, which are equal to the velocity of the background horizontal wind at the top boundary of the convective layer of 3-4 km height. It seems that the background horizontal wind in which the convection is embedded determines the phase velocity of them.

Substituting $\bar{u}(z), N^{2}(z)$ and $\bar{T}=250 \mathrm{~K}$ into (8), vertical profiles of square of horizontal wavenumber $k^{2}$ associated with the waves of $m^{2}=0$ for $c=5 \mathrm{~m} \mathrm{~s}^{-1}, c=10 \mathrm{~m} \mathrm{~s}^{-1}$ and $c=2.5 \mathrm{~m} \mathrm{~s}^{-1}$ are shown in Fig. 10. The left side of lines means that the waves become internal and the right side means that the waves cannot propagate vertically. It is found that the values of $k^{2}$ for $c=10 \mathrm{~m} \mathrm{~s}^{-1}$ at $9.6 \mathrm{~km}$ height indicates that the smallest wavelength is about $10 \mathrm{~km}$ with which the wave can propagate vertically. This figure shows that the wave with the horizontal wavelength of the order of $10 \mathrm{~km}$ is chosen and shorter than that is filtered out by the background westerly. As the convection becomes more active with time, the level of the strongest disturbance in the convective layer is getting higher. The velocity of the background wind is larger with height in the convective layer, so that the horizontal phase velocity of the radiated dominant IGWs is getting faster. According to Fig. 10, it is found that the smallest wavelength of which the wave is internal becomes larger when the phase velocity gets faster.

Larsen et al. (1982) observed stratospheric wave motions above tropospheric convections, and found a high frequency wave with a period of $6 \mathrm{~min}$ and the vertical wavelength of 7 $\mathrm{km}$. The dominant waves in the present model simulation are consistent with these high frequency IGWs observed in the real atmosphere in spite of the difference of the convection mechanism.

\subsection{IGWs-critical layer interaction and secondary wave radiation by critical layer instability}

Figure 11 shows the vertical profile of vertical wind at $x=15 \mathrm{~km}$ at the time of $4,800 \mathrm{~s}$. Figures 7 and 11 show that the wave amplitude decreases and the vertical wavelength becomes short and the phase line approaches horizontal above $20 \mathrm{~km}$. As mentioned above, this behavior is due to the critical layer interaction found by Booker and Bretherton (1967). As the time goes on, the critical layer structure becomes more complicated in the range from $18 \mathrm{~km}$ to $24 \mathrm{~km}$ height. Figure 12 shows the cross section of vertical wind below $60 \mathrm{~km}$ height at the time steps of $13,600 \mathrm{~s}, 14,400 \mathrm{~s}$, and $15,200 \mathrm{~s}$. At these time steps, it is found that the convection becomes more active, and the maximum vertical wind in the lower tropospheric convection exceeds $4 \mathrm{~m} \mathrm{~s}^{-1}$ and the amplitude of the IGW generated by the convection also reaches above $2 \mathrm{~m} \mathrm{~s}^{-1}$ above $16 \mathrm{~km}$ height. The wave pattern becomes random around the critical layer. It is found that the new eastward tilted wave pattern is propagating upward (the phase is moving downward and eastward) above the critical layer. These distributions show that the critical layer instability of the incident IGWs and the secondary wave radiation by the instability.

Figure 13(a) shows the deviation of the zonal mean wind from the initial basic state. There is a deceleration region below $23 \mathrm{~km}$ height and the strongest deceleration occurs at $19 \mathrm{~km}$ height. The relationship between the zonal mean wind $\bar{U}(z, t)$ and the momentum flux is given as follows,

$$
\frac{\partial \bar{U}(z, t)}{\partial t} \approx \frac{-1}{\rho_{\mathrm{B}}(z)} \frac{\partial\left(\rho_{\mathrm{B}} \overline{u^{\prime} w^{\prime}}\right)}{\partial z},
$$

where $u^{\prime}$ denotes the deviation from the zonal mean wind, $w^{\prime}$ the vertical wind and overbar denotes the zonal mean.

Figure 13(b) shows the vertical profile of the momentum 


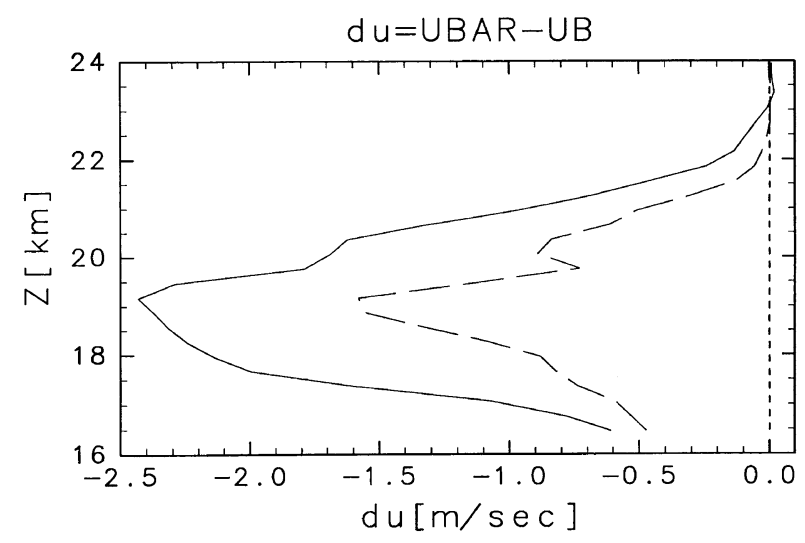

(a)

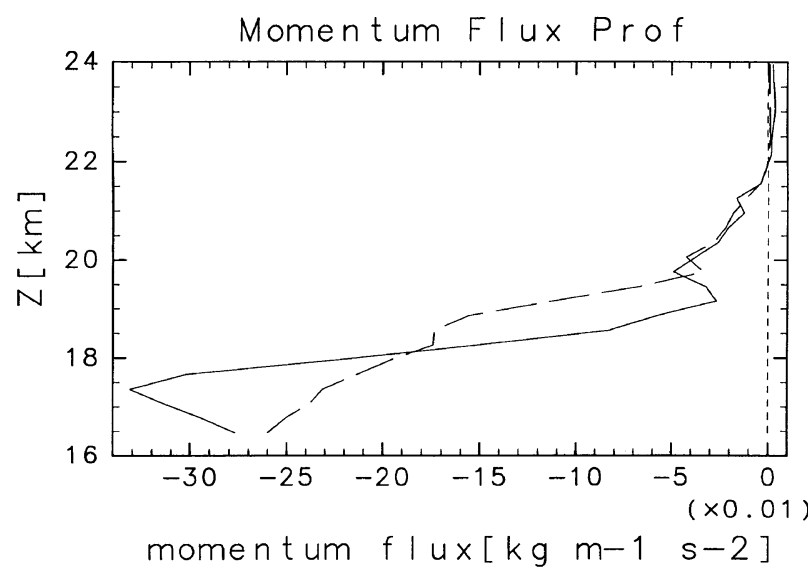

(b)

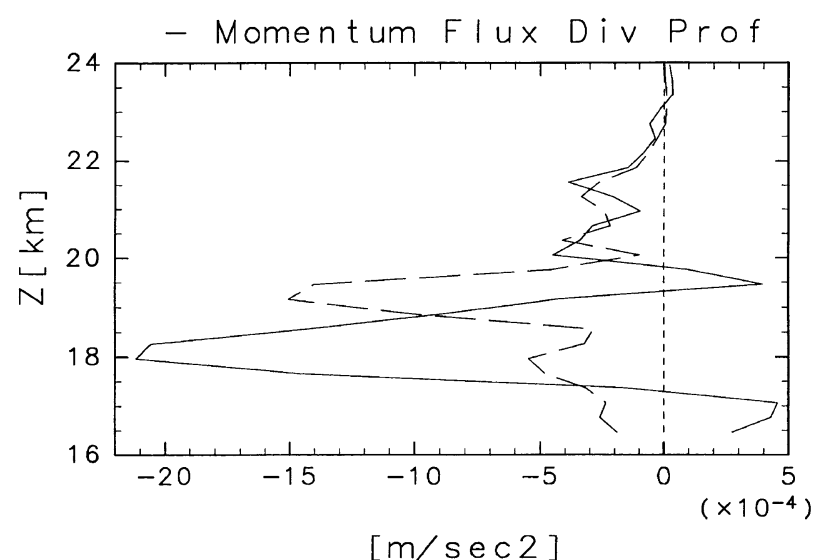

(c)

Fig. 13. Vertical profiles of (a) deviation of horizontal wind from the initial background horizontal wind $U_{\mathrm{B}}(z)$, (b) momentum flux, and (c) inverse sign of vertical divergence of the momentum flux. Dotted, dashed, and solid lines show the values at 5,600 s, 13,600 s, and 15,200 s, respectively.

flux. It is found that the momentum flux decreases abruptly from $18 \mathrm{~km}$ to $22 \mathrm{~km}$ height. Figure 13(c) shows the momentum flux divergence of RHS of (9). The peak value is

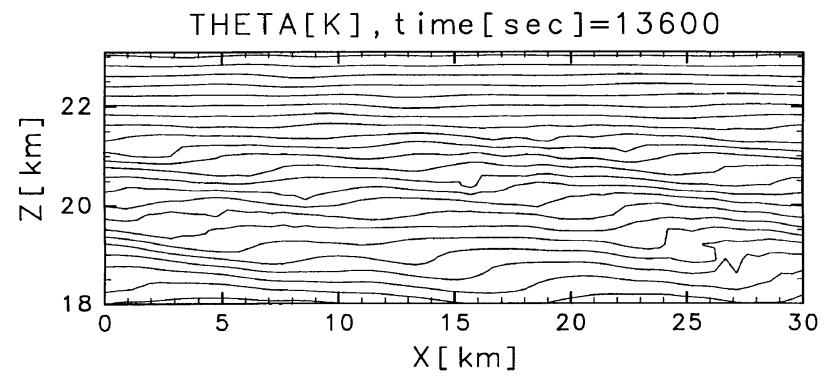

CONTOUR INTERVAL $=6.000 E+00$

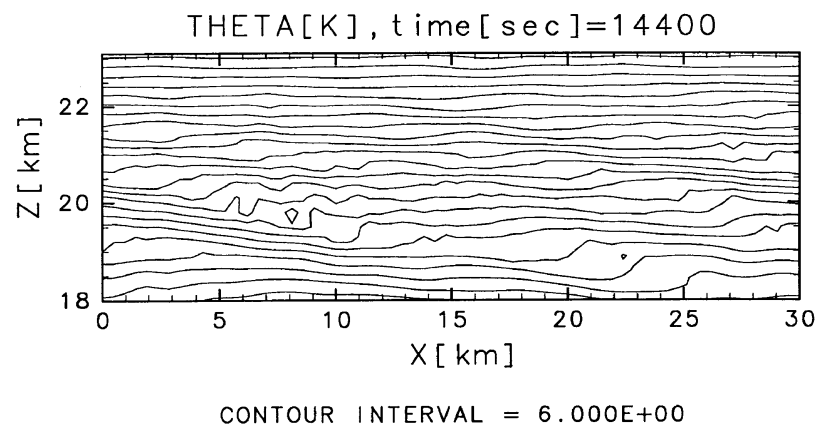

THETA $[K], t$ ime $[$ sec $]=15200$

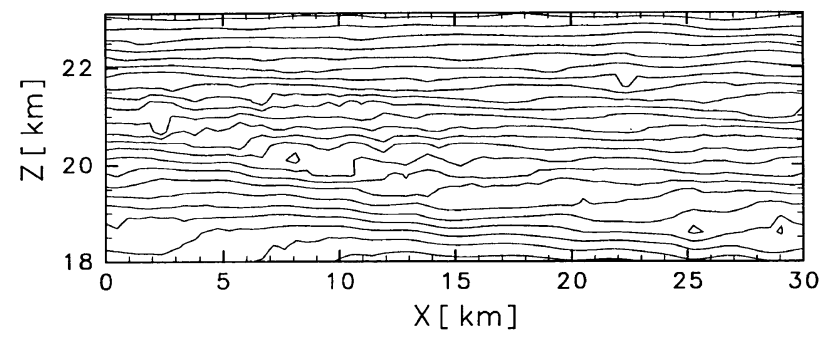

CONTOUR INTERVAL $=6.000 E+00$

Fig. 14. Contour plots of potential temperature $(\mathrm{K})$ at the time steps of $13,600 \mathrm{~s}, 14,400 \mathrm{~s}$, and $15,200 \mathrm{~s}$.

$-2.1 \times 10^{-3} \mathrm{~m} \mathrm{~s}^{-2}\left(-180 \mathrm{~m} \mathrm{~s}^{-1} \mathrm{day}^{-1}\right)$. There is some regions where the RHS of (9) is positive. It is inferred to be brought by a transient IGW passing in the form of a wavepacket. As mentioned above, the typical phase velocity of the IGWs generated by the convection is about $10 \mathrm{~m} \mathrm{~s}^{-1}$ and the zonal mean wind velocity at about $20 \mathrm{~km}$ height is equal to this phase velocity, so that it is found that the horizontal momentum deposition due to the IGWs-critical layer interaction brings this deceleration of the zonal mean wind.

To realize the closing structure of the tropospheric jet in the wintertime hemisphere in a GCM, gravity wave drag paramererization is used, and a magnitude of deceleration near $20 \mathrm{~km}$ height is given about $1 \mathrm{~m} \mathrm{~s}^{-1}$ day $^{-1}$ (e.g., McFarlane, 1987). Our result of the critical layer deceleration is two order larger than that value. It is noted that our simulation assumed the continuous convective instability in both the time and space, but it might be sparse in the real 


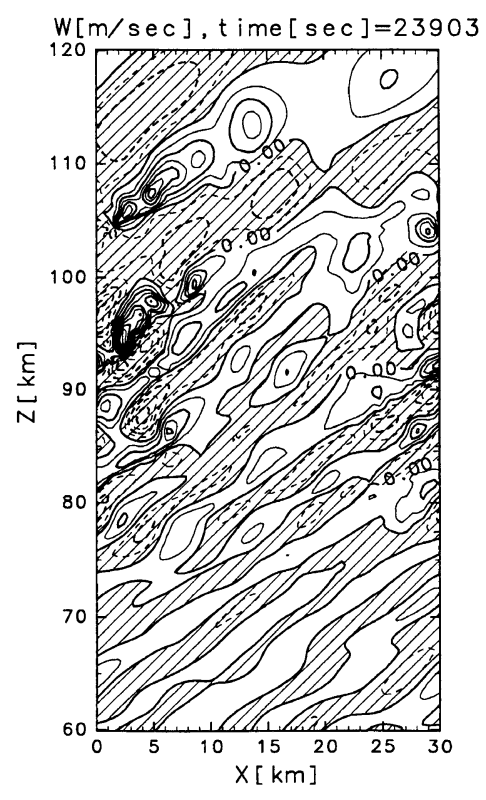

CONTOUR INTERVAL $=5.000 E+00$

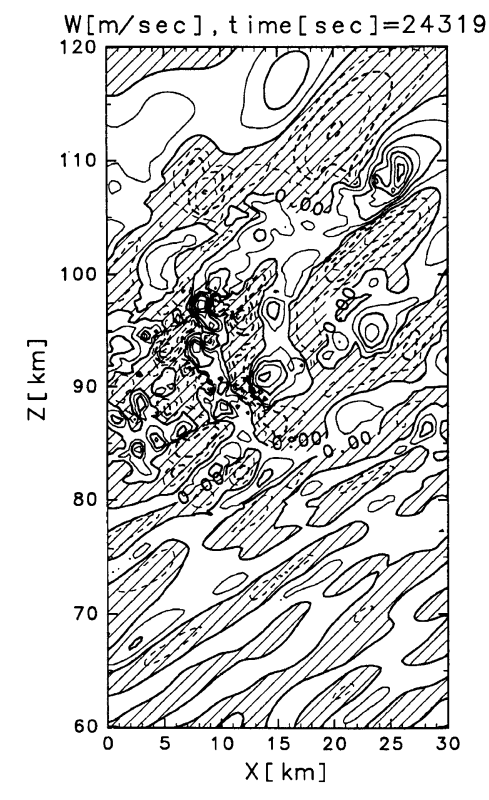

CONTOUR INTERVAL $=5.000 E+00$

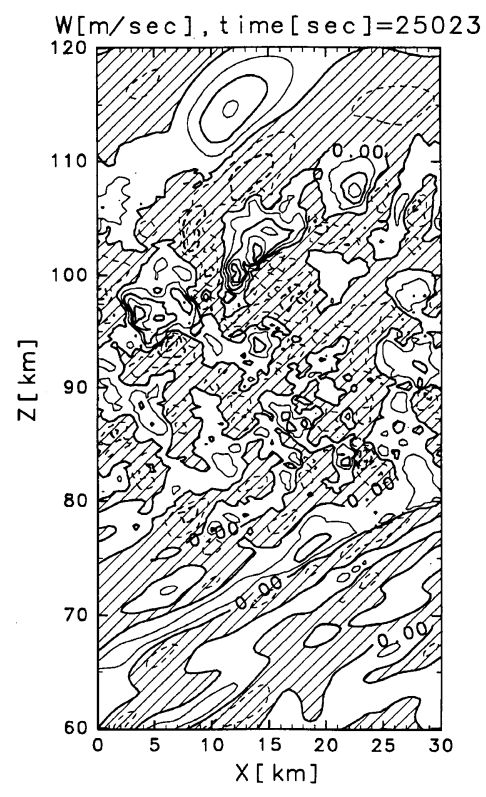

CONTOUR INTERVAL $=5.000 E+00$

Fig. 15. Contour plots of vertical wind $w\left(\mathrm{~m} \mathrm{~s}^{-1}\right)$ at the time steps of $23,903 \mathrm{~s}, 24,319 \mathrm{~s}$, and $25,023 \mathrm{~s}$.

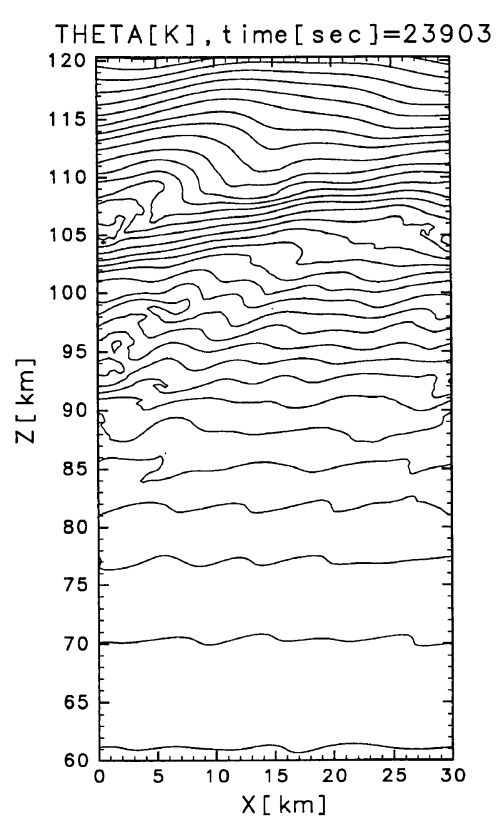

CONTOUR INTERVAL $=1.000 E+03$

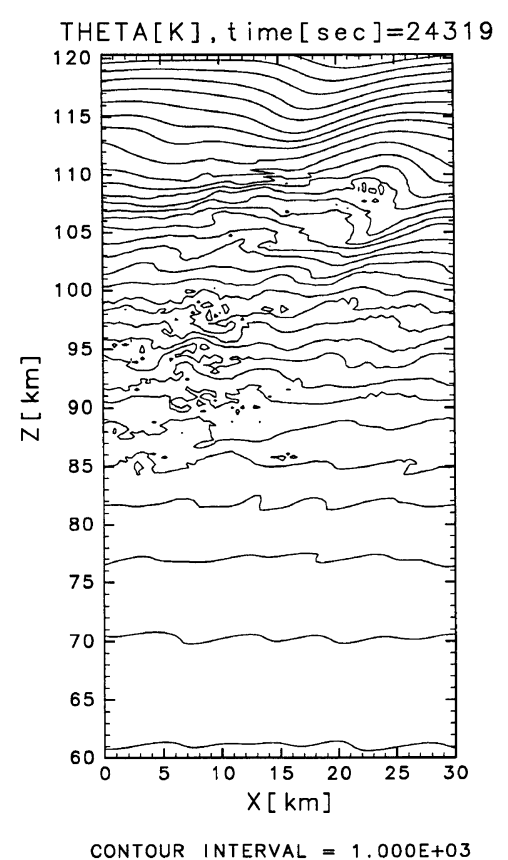

CONTOUR INTERVAL $=1.000 E+03$

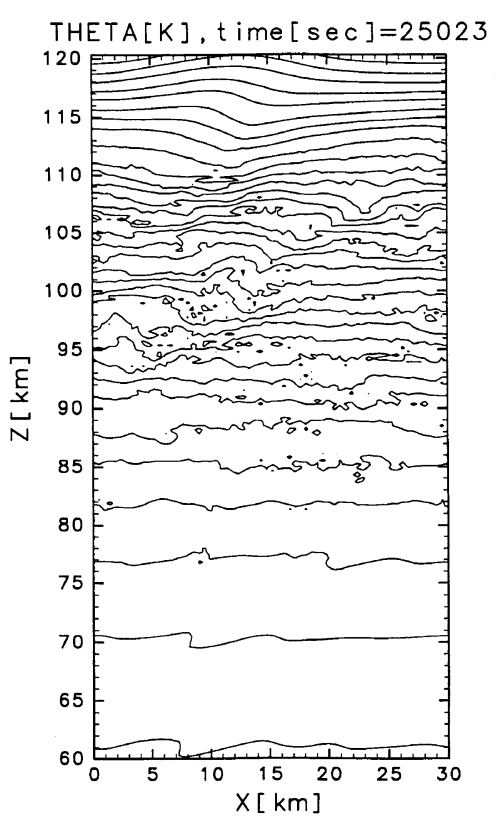

CONTOUR INTERVAL $=1.000 E+03$

Fig. 16. As in Fig. 15, except for the potential temperature.

atmosphere. Thus intermittent structure may reduce the net momentum flux in the real atmosphere.

Figure 14 shows the contour plots of the potential temperature from $18 \mathrm{~km}$ to $22.5 \mathrm{~km}$ height. Local overturning of the isentropic lines is seen and it shows the convective instability of the incident waves around the critical layer. This instability generates the random pattern of the critical layer and radiates secondary waves. The space-time spectral analysis for the time span from $13,600 \mathrm{~s}$ to $15,648 \mathrm{~s}$ at $30 \mathrm{~km}$ height indicates some spectral peaks at eastward moving waves with horizontal wavelength $\lambda_{\mathrm{H}} 5 \mathrm{~km}$, period $\tau 5.7 \mathrm{~min}$, and phase speed $c 15 \mathrm{~m} \mathrm{~s}^{-1}$ (referred as k6), with $\lambda_{\mathrm{H}} 6 \mathrm{~km}, \tau 8.5 \mathrm{~min}$, and $c 12 \mathrm{~m} \mathrm{~s}^{-1}(\mathrm{k} 5)$, and with $\lambda_{\mathrm{H}} 30 \mathrm{~km}, \tau 11 \mathrm{~min}$, and c $45 \mathrm{~m} \mathrm{~s}^{-1}(\mathrm{k} 1)$, but there is also some westward moving waves of which amplitude is very small. The vertical wavelength of both of $\mathrm{k} 6$ and $\mathrm{k} 5$ is deduced to be about $7 \mathrm{~km}$ and 

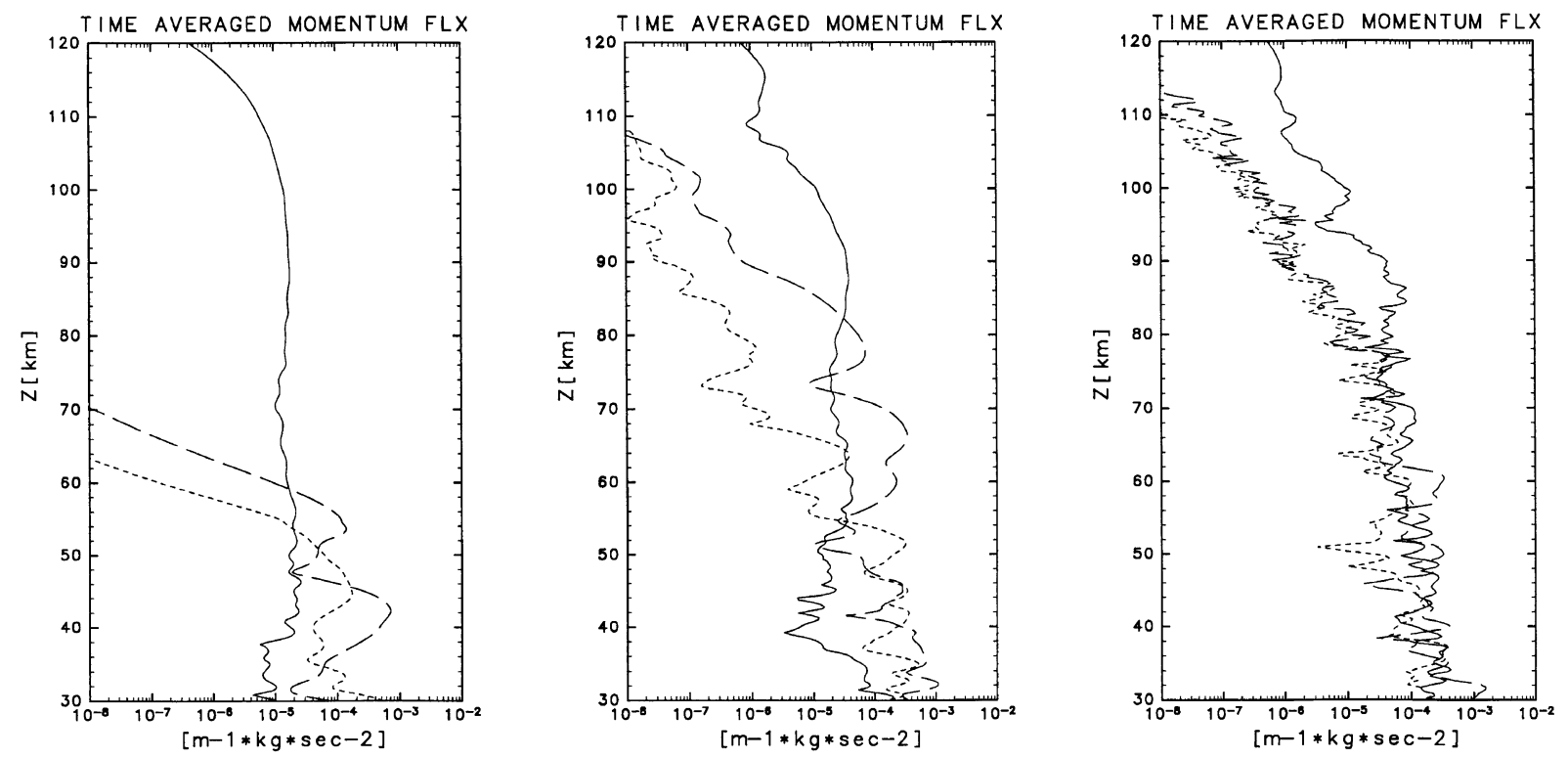

Fig. 17. Vertical profiles of momentum flux time-averaged over 7.5 min interval associated with the wave K1 (solid line), K5 (dashed line) and K6 (dotted line). Time average is done over $18,016 \mathrm{~s}-18,464 \mathrm{~s}, 21,984 \mathrm{~s}-22,432 \mathrm{~s}$, and $25,536 \mathrm{~s}-25,984 \mathrm{~s}$.

the vertical wavelength of $\mathrm{k} 1$ is also deduced to be $13 \mathrm{~km}$. Doppler-shift is negligible above $24 \mathrm{~km}$ height because there are no zonal mean winds. These parameters of the waves satisfy the dispersion relation (8), so that secondary radiated waves are also identified as IGWs. As the case of IGWs generation by the convection, the zonal mean wind velocity of the secondary wave source region determines the phase velocity of the secondary wave. However the wave $\mathrm{k} 1$ is not the case and the phase velocity is much faster than the zonal wind velocity. It is not cleared the generation mechanism of the IGW with fast phase velocity. In the unstabilized critical layer, the nonlinear interaction becomes strong, then it might be possible to generate the wave $\mathrm{k} 1$ by the nonlinear interactions. But the present work is focusing on the propagation of IGWs throughout the middle atmosphere, so that we do not deeply investigate the generation mechanism. It will be done in a separate paper.

\subsection{IGWs breakdown in the upper middle atmosphere}

Figure 15 shows that the contour plots of vertical wind from $60 \mathrm{~km}$ to $120 \mathrm{~km}$ height at the time steps of 23,903 s, 24,319 s, and 25,023 s after about 3 hours shown in Fig. 12. Figure 16 shows that the contour plots of potential temperature at the same time steps. It is found that the secondary IGWs radiated by the critical layer instability in the lower stratosphere break in the upper mesosphere and the breaking region spread downward with the time goes on, and turbulent layer is generated. It is also found that the IGWs propagates vertically in the form of wavepackets and break by local convective in stability due to the exponential growth of the wave amplitude with height. In the previous section, it is shown that the main IGWs generated by the critical layer instability are the eastward moving waves $\mathrm{k} 5, \mathrm{k} 6$ and $\mathrm{k} 1$. The vertical space spectral analysis, which is conducted using the data from $40 \mathrm{~km}$ to $78 \mathrm{~km}$, the waves with zonal wavenumbers $k=5, k=6$ and $k=1$ have representative vertical wave- length $\lambda_{\mathrm{V}} 6 \mathrm{~km}, 6 \mathrm{~km}$, and $13 \mathrm{~km}$, respectively. The group velocity $c_{\mathrm{gz}}$ of an IGW is given by the following equation;

$$
c_{\mathrm{gz}}=\frac{-N m|k|}{\left(k^{2}+m^{2}+\frac{1}{4 H^{2}}\right)^{3 / 2}} .
$$

For example, $c_{\mathrm{gz}}$ of the dominant wave $\mathrm{k} 5$ and $\mathrm{k} 6$ calculated by (10) assuming $N=2.0 \times 10^{-2} \mathrm{~s}^{-1}$ and $\mathrm{H}=7.0 \mathrm{~km}$ is $7.0 \mathrm{~m} \mathrm{~s}^{-1}$ and $6.0 \mathrm{~m} \mathrm{~s}^{-1}$, respectively. Figure 17 shows vertical profiles of momentum flux time-averaged over 7.5 min interval associated with the waves which are specified only with the zonal wavenumber $k=5, k=6$ and $k=1$ (hereafter, those waves are referred as K5, K6 and K1). The momentum fluxes associated with these waves show the vertical propagation of these waves with different vertical group velocity. $c_{\mathrm{gz}}$ of $\mathrm{K} 5$ and $\mathrm{K} 6$ waves estimated from Fig. 17 is about $8 \mathrm{~m} \mathrm{~s}^{-1}$. Figure 17 shows that the wave type K5 and K6 simultaneously propagates vertically with almost the same group velocity. This is consistent with the result of the sub-section that $\mathrm{k} 5$ and $\mathrm{k} 6$ waves are simultaneously radiated from the critical layer. $c_{\mathrm{gz}}$ of $\mathrm{k} 1$ estimated by (10) is $13 \mathrm{~m} \mathrm{~s}^{-1}$ and it is found that the wave $\mathrm{K} 1$ is already radiated at the time step of $9,024 \mathrm{~s}$, and this is earlier than the radiation of the waves K5 and K6, (See Fig. 8). The first breakdown is due to the wave $\mathrm{K} 1$ around $90 \mathrm{~km}$ height and the waves $\mathrm{K} 5$ and K6 break down in the lower height after the first breakdown.

Figures 18 (a), (b), and (c) show vertical profiles of the deviation from the initial horizontal wind, horizontal average of momentum flux and vertical divergence of the momentum flux, respectively. Solid lines indicate the time-averaged data over 23,552-25,984 $\mathrm{s}$ when the breakdown is occurring. Dotted and dashed lines indicate snapshots of 21,120 s and $23,520 \mathrm{~s}$ when it is the time of the beginning of the breakdown and the middle phase on the breakdown, respectively. It is found that westerly wind is induced due to the breakdown of the IGWs. It is also found that the momentum flux 


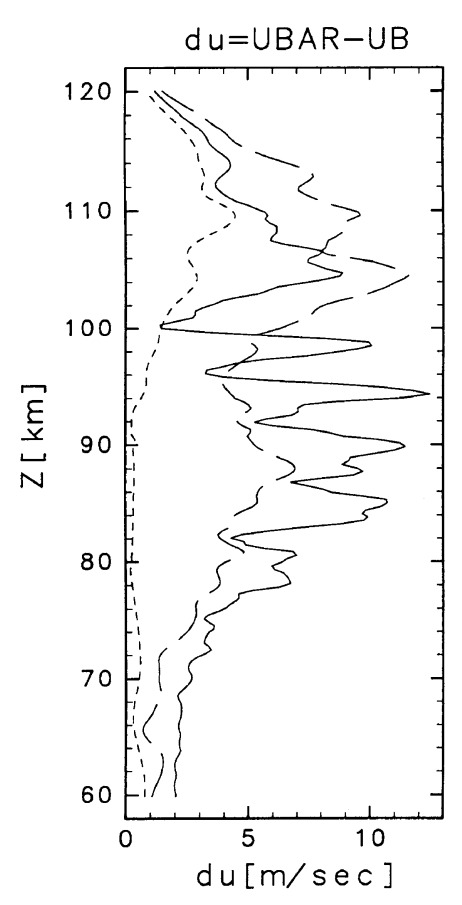

(a)

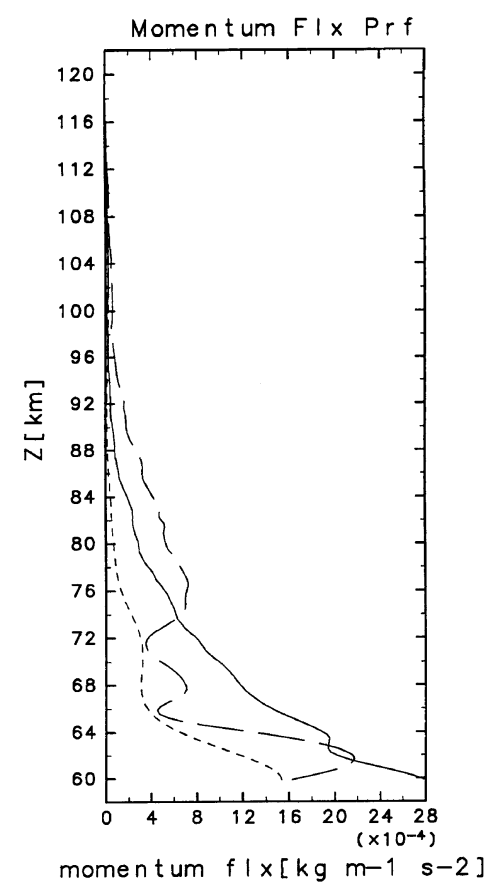

(b)

Fig. 18. Vertical profiles of (a) deviation of horizontal wind from the initial background horizontal wind $U_{\mathrm{B}}(z)$, (b) momentum flux, and (c) inverse sign of vertical divergence of the momentum flux. Dotted, dashed, and solid lines show the values at $21,120 \mathrm{~s}, 23,520 \mathrm{~s}$, and time-averaged over $23,552 \mathrm{~s}-25,984 \mathrm{~s}$, respectively.

decreases with height and the westerly acceleration occurs in the upper mesosphere and lower thermosphere, except for some irregularities. The instantaneous acceleration of the

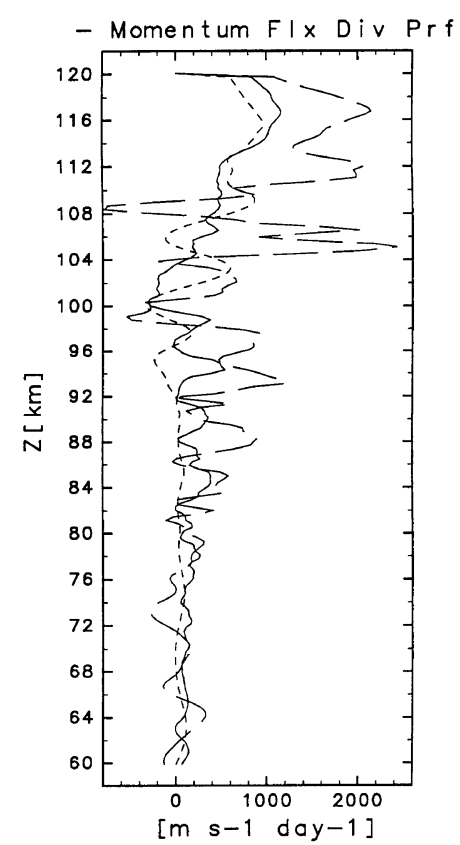

(c)

Fig. 18. (continued).

zonal mean wind due to the breakdown is the order of $10^{3}$ $\mathrm{m} \mathrm{s}^{-1} \mathrm{day}^{-1}$ at $93 \mathrm{~km}$ as indicated by the dashed line, but the height and time-averaged acceleration is of the order of $10^{2}$ $\mathrm{m} \mathrm{s}^{-1} \mathrm{day}^{-1}$, which is the same order of theoretical estimations and observational reports about the momentum flux in this region (e.g., Lindzen, 1981; Fritts and Vincent, 1987). It is noted that our simulation assumed the continuous convective instability in both the time and space, so that the momentum flux might be much larger than that induced by convectively generated IGWs in the real atmosphere, and the deceleration in the real atmosphere cannot be attributed to the gravity waves generated by the present mechanism.

Figures 19 (a) and (b) show the power spectra of the horizontal and vertical wind in the wavenumber space $m$ between $80 \mathrm{~km}$ and $118 \mathrm{~km}$ heights, respectively. It is found that the power spectra show approximate $m^{-3}$ dependence at higher wavenumber region after the waves are breakdown $(t=24,320 \mathrm{~s})$. Such dependence has been reported by VanZandt (1982) for the horizontal wind spectra, and it is also reported for the vertical wind spectra by Fritts and Hoppe (1995). It is predicted that the dependence $m^{-3}$ is due to the wave saturation and breakdown (Dewan and Good, 1986), and it is shown that such the universal power law is also realized after the wave breaking in the present model.

\section{Conclusions and Remarks}

Generation of IGWs by the tropospheric convections and the vertical propagation of the generated IGWs throughout the middle atmosphere are simulated using the nonhydrostatic compressible nonlinear two-dimensional numerical model. It is found that IGWs are generated by the tropospheric convections. It is also found that the secondary 


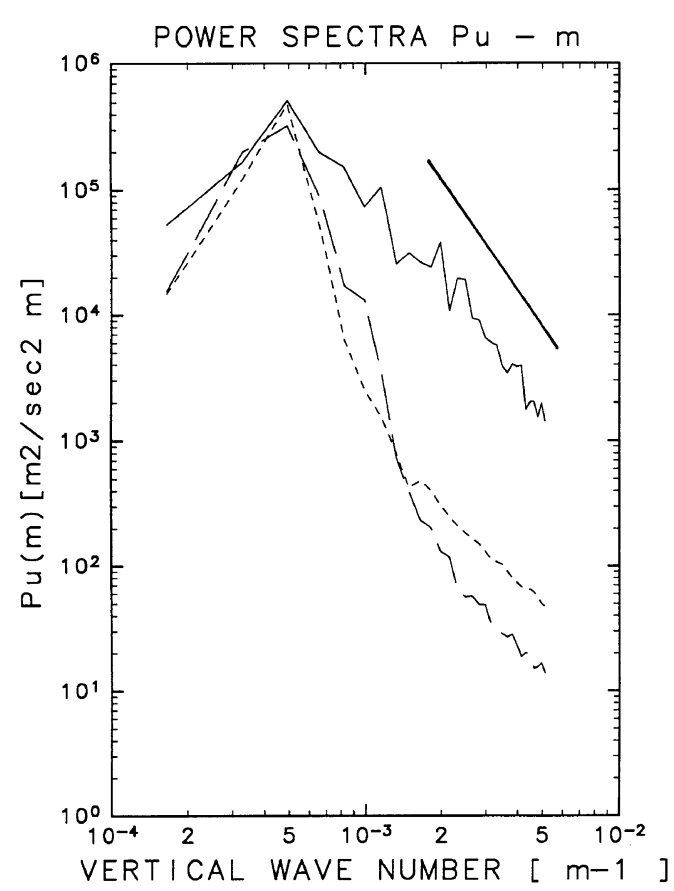

(a)

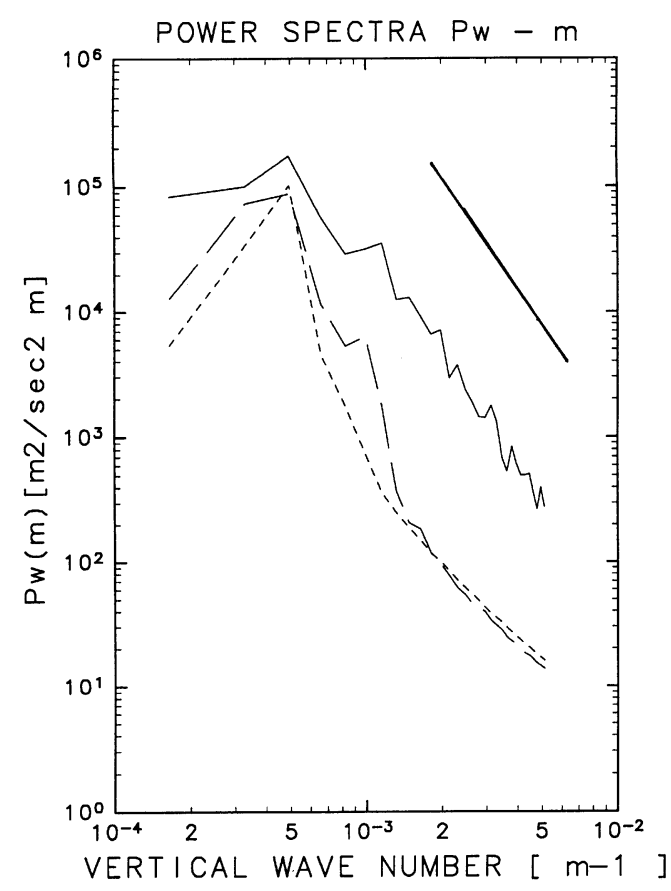

(b)

Fig. 19. Power spectra versus vertical wavenumber $m$ at between $80 \mathrm{~km}$ and $118 \mathrm{~km}$, (a) for the horizontal velocity deviation from the zonal mean, and (b) for the vertical velocity. Dotted, dashed, and solid lines show the values at 19,840 s, 21,440 s, and 24,320 s, respectively. Thick solid line has a slope of $m^{-3}$.

IGWs are radiated from the critical layer through the critical layer instability, and these waves breakdown and accelerate the zonal mean wind in the upper middle atmosphere due to local convective instability because of the exponential growth of the amplitude with height.

In the present work, the followings are demonstrated:

It is realized that the first IGWs have small wavelength of about $10 \mathrm{~km}$ with the aspect ratio $\mathrm{k} / \mathrm{m}$ of about 1 and short period of about $10 \mathrm{~min}$. It is found that this fact results from the process that the waves with the smallest horizontal wavelength which can propagate vertically in the prescribed westerly is selected. The background zonal mean wind in the convective layer determines the phase velocity of the dominant IGWs in both cases of the convective wave generation and the critical layer wave generation. However, in the secondary IGWs radiated from the unstabilized critical layer, the wave with the phase velocity much faster than the background zonal mean wind velocity is also seen.

The deceleration due to the IGWs-critical layer interaction is instantaneously marked by $-180 \mathrm{~m} \mathrm{~s}^{-1} \mathrm{day}^{-1}$. This value is much larger than the value due to gravity wave parameterization introduced to some general circulation models to realize the weak zonal mean winds in the lower stratosphere (e.g., McFarlane, 1987). However the present results are not surprising, because the present model always generates gravity waves keeping the convectively unstable situation. In the realistic situation, gravity waves may be more sparse both in the space and the time.

The IGWs generated by the critical layer instability begin to break in the upper mesosphere and the breakdown region spreads down to the lower mesosphere atmosphere with time. The first propagating wave is $\lambda_{\mathrm{H}} 30 \mathrm{~km}(\mathrm{~K} 1)$, and the next propagating waves are $\lambda_{\mathrm{H}} 6 \mathrm{~km}$ and $5 \mathrm{~km}(\mathrm{~K} 5$ and $\mathrm{K} 6$, respectively). $\mathrm{K} 1$ breaks in the upper level, and $\mathrm{K} 5$ and $\mathrm{K} 6$ breaks in the lower level. The breakdown strongly depends on the exponential growth of the amplitude with height. The difference of the breaking level depends on the amplitudes of the wave and the vertical wavelength. The waves $\mathrm{K} 5$ and $\mathrm{K} 6$ have larger amplitude and shorter vertical wavelength than the wave $\mathrm{K} 1$, so that the breaking level is lower than that of K1. The magnitude of acceleration in the IGWs breaking region is about $100 \mathrm{~m} \mathrm{~s}^{-1} \mathrm{day}^{-1}$, which has same order magnitude with the momentum deposition by theoretical estimations and observational reports (e.g., Lindzen, 1981; Fritts and Vincent, 1987). It is noted that our simulation assumed the continuous convective instability in both the time and space, so that the momentum flux might be much larger than that induced by convectively generated IGWs in the real atmosphere, and not all the deceleration in the real atmosphere can be attributed to the gravity waves generated by the present mechanism. It has been considered that IGWs are important source of momentum in the upper middle atmosphere and they propagate directly from the troposphere without breakdown on the way. However the present result suggests the importance of the secondary generated waves due to the breakdown of the primary waves on the way.

The power spectra of the wind fluctuation shows $m^{-3}$ dependence on the vertical wavelength in the upper mesospheric breaking region. The universal power law which is due to the wave saturation and breakdown is observed in the real atmosphere and theoretically predicted (VanZandt, 1982; Dewan and Good, 1986).

The simulations under the more realistic zonal mean wind 
are now conducted, and the results will be shown in a separate paper.

Acknowledgments. The authors wish to express their thanks to Drs. T. Hirooka, K. Sato, K. Nakajima, Y. Miyoshi, and T. Iwayama for their valuable comments and suggestions. The GFD-DENNOU Library was used for drawing the figures.

\section{References}

Alexander, M. J., J. R. Holton, and D. R. Durran, The gravity wave response above deep convection in a squall line simulation, J. Atmos. Sci., 52, 2212-2226, 1995.

Asai, T., Stability of a plane parallel flow with variable vertical shear and unstable stratification, J. Meteor. Soc. Japan., 48, 129-139, 1970.

Booker, J. R. and F. P. Bretherton, The critical layer for internal gravity waves in a shear flow, J. Fluid. Mech., 27, 513-539, 1967.

Bretherton, F. P., The propagation of groups of internal gravity waves in a shear flow, Q. J. R. Meteorol. Soc., 92, 466-480, 1966.

Dewan, E. M. and R. E. Good, Saturation and the "universal" spectrum for vertical profiles of horizontal scalar winds in the atmosphere, J. Geophys. Res., 91, 2742-2748, 1986.

Forvell, R. G., D. Durran, and J. R. Holton, Numerical simulations of convectively generated stratospheric gravity waves, J. Atmos. Sci., 49, 14271442, 1992.

Fritts, D. C., The excitation of radiating waves and Kelvin-Helmholtz instabilities by the gravity wave-critical level interaction, J. Atmos. Sci., 36 , 12-23, 1979.

Fritts, D. C., The transient critical-level interaction in a Boussinesq fluid, $J$. Atmos. Sci., 87, 7997-8016, 1982.

Fritts, D. C. and U. P. Hoppe, High-resolution measurements of vertical velocity with the European incoherent scatter VHF radar 2: Spectral observations and model comparisons, J. Geophys. Res., 100, 16827$16838,1995$.

Fritts, D. C. and G. D. Nastrom, Sources of mesoscale variability of gravity waves II: Frontal, convective, and jet stream excitation, J. Atmos. Sci., 49, 111-127, 1992.

Fritts, D. C. and R. A. Vincent, Mesospheric momentum flux studies at
Adelaide, Australia: observations and a gravity wave-tidal interaction model, J. Atmos. Sci., 44, 605-619, 1987.

Fritts, D. C., J. R. Isler, and Ø. Andreassen, Gravity wave breaking in two and three dimensions 2: Three-dimensional evolution and instability structure, J. Geophys. Res., 99, 8109-8123, 1994.

Geller, M. A., H. Tanaka, and D. C. Fritts, Production of turbulence in the vicinity of critical levels for internal gravity waves, J. Atmos. Sci., 32, 2125-2135, 1975.

Hodges, R. R., Jr., Generation of turbulence in the upper atmosphere by internal gravity waves, J. Geophys. Res., 72, 3455-3458, 1967.

Larsen, M. F., W. E. Swartz, and R. F. Woodman, Gravity-wave generation by thunderstorms observed with a vertically-pointing $430 \mathrm{MHz}$ radar, Geophys. Res. Lett., 9, 571-574, 1982.

Lindzen, R. S., Turbulence and stress owing to gravity wave and tidal breakdown, J. Geophys. Res., 86, 9707-9714, 1981.

Matsuno, T., A quasi one-dimensional model of the middle atmosphere circulation interacting with internal gravity waves, J. Meteor. Soc. Japan., 60, 215-226, 1982.

McFarlane, N. A., The effect of orographically excited gravity wave drag on the general circulation of the lower stratosphere and troposphere, $J$. Atmos. Sci., 44, 1775-1800, 1987.

Palmer, T. N., G. J. Shutts, and R. Swinbank, Alleviation of a systematic westerly bias in general circulation and numerical weather prediction models through an orographic gravity wave drag parameterization, $Q . J$. R. Meteorol. Soc., 112, 10001-10039, 1986.

Prusa, J. M., P. K. Smolarkiewicz, and R. R. Garcia, Propagation and breaking at high altitudes of gravity waves excited by tropospheric forcing, $J$. Atmos. Sci., 53, 2186-2216, 1996.

Sato, K., Small-scale wind disturbances observed by the MU Radar during the passage of typhoon Kelly, J. Atmos. Sci., 50, 518-537, 1993.

Sato, K., Observational studies of gravity waves associated with convection, Gravity Wave Processes, in NATO ASI Series, edited by K. Hamilton, pp. 63-68, Springer, 1997.

VanZandt, T. E., A universal spectrum of buoyancy waves in the atmosphere, Geophys. Res. Lett., 9, 575-578, 1982.

K. Goya (e-mail: goya@rossby.geo.kyushu-u.ac.jp) and S. Miyahara 